\title{
Performance comparison human activity recognition using simple linear method
}

\author{
Wahyu Andhyka Kusuma ${ }^{1}$, Zamah Sari ${ }^{2}$, Agus Eko Minarno ${ }^{3}$, Hardianto Wibowo ${ }^{4}$, Denar Regata Akbi $^{\star 5}$, Naser \\ Jawas $^{6}$ \\ Universitas Muhammadiyah Malang, Indonesia ${ }^{1,2,3,4,5}$ \\ University of Warwick, England ${ }^{6}$
}

\section{Article Info}

Keywords:

Human Activity Recognition, Physical

Analysis, Linear Method

Article history:

Received 26 December 2019

Revised 07 January 2020

Accepted 18 January 2020

Published 06 February 2020

Cite:

Kusuma, W., Sari, Z., Minarno, A., Wibowo, H., Akbi, D., \& Jawas, N. (2020). Performance Comparisson Human Activity Recognition using Simple Linear Method. Kinetik: Game Technology, Information System, Computer Network, Computing, Electronics, and Control, 5(1).

doi:https://doi.org/10.22219/kinetik.v5i1.1025

${ }^{*}$ Corresponding author.

Denar Regata Akbi

E-mail address:

dnarregata@umm.ac.id

\begin{abstract}
Human activity recognition (HAR) with daily activities have become leading problems in human physical analysis. HAR with wide application in several areas of human physical analysis were increased along with several machine learning methods. This topic such as fall detection, medical rehabilitation or other smart appliance in physical analysis application has increase degree of life. Smart wearable devices with inertial sensor accelerometer and gyroscope were popular sensor for physical analysis. The previous research used this sensor with a various position in the human body part. Activities can classify in three class, static activity (SA), transition activity (TA), and dynamic activity (DA). Activity from complexity in activities can be separated in low and high complexity based on daily activity. Daily activity pattern has the same shape and patterns with gathering sensor. Dataset used in this paper have acquired from 30 volunteers. Seven basic machine learning algorithm Logistic Regression, Support Vector Machine, Decision Tree, Random Forest, Gradient Boosted and K-Nearest Neighbor. Confusion activities were solved with a simple linear method. The purposed method Logistic Regression achieves $98 \%$ accuracy same as SVM with linear kernel, with same result hyperparameter tuning for both methods have the same accuracy. LR and SVC its better used in SA and DA without TA in each recognizing.
\end{abstract}

\section{Introduction}

Human activity recognition (HAR) aiming to identify the actions given by body pattern with a set of observation in several sequence activity. Nowadays, HAR has a field of topic study that increased several interests in many areas. Due to its wide application in human activities behavior, living assistance, home applied, security, pedestrian tracking, medical, rehabilitation, elderly and disability and wider to smart cities and transportation topics. From several topics in HAR, the most important research topic in this area within activity detection due to its implementation in human computer interaction $(\mathrm{HCl})$, medical, rehabilitation, and another implementation area [1]. Previous research on the medical and rehabilitation topic was increased significantly. HAR has improved health status for diabetic patient, elderly monitoring, non-communicable disease (NCD), calories [2][3] and fall detection [4], human behavior [5]. HAR requires a robust technique. HAR must be detected simple and complex activity especially that can be used to detect under free daily motion, for example, recognition of fall detection, especially in elderly fall detection. Fall injuries caused an inability to live independently in a broader impact lead life-threatening [6].

HAR has improved life quality like an obese sufferer. Obesity is a non communicable disease (NCD) that's mean that not contagious is a preventable disease. Obesity has several factors, including education, behavior change, personality [7]. Poor diet, less movement, an increase of weight and body mass index (BMI) as an aspect that causes premature physical deterioration and cognitive decline [8][9][10]. Lifestyle changes and awareness of it are necessary, activity recognition as a technology for monitoring and improvement can suggest any information may be needed for it.

Detecting HAR accurately and in real time is a challenging problem for several reasons [1]. The rapid development of machine learning also increases in HAR field. There are several methods to perform activity recognition: using multimedia (video), wearable devices (smart phone, smart watch) and ambient sensing [1][11]. Several approaches were recognizing activities from one [12][13][14][15][16] or more sensor [10][17][18] placement at the human body. Another approach based on vision was used in previous research but with some limitations, such an environmental restriction [14]. The camera amount used as the sensor was created a dimension of image as data. In other, placement sensors as wearable devices have more reliable to measure an evaluation based on pattern activities.

HAR with wearable devices provide data from the movement of body, temperature, heart rate. Popular sensor where was used several authors used the accelerometer and gyroscope (inertial sensor). Single or combine that sensor make several opportunities for research. Both accelerometer and gyroscope have three axial axes it presents several

Cite: Kusuma, W., Sari, Z., Minarno, A., Wibowo, H., Akbi, D., \& Jawas, N. (2020). Performance Comparisson Human Activity Recognition using Simple Linear Method. Kinetik: Game Technology, Information System, Computer Network, Computing, Electronics, and Control, 5(1). doi:https://doi.org/10.22219/kinetik.v5i1.1025 
combinations both them. The main areas to find less complexity especially as an embedded system or applied as the mobile application. HAR with a wearable-based system including smart phone is that the activity detection can be personalized for specific person [19] and for specific body pattern. However, the accuracy of inertial sensors based on accurate signal processing recognizing pattern activities was becoming problems in the HAR field.

Previous research recognizing several activities from a different kind of sensor, except camera and accelerometer, gyroscope sensor other author used electromyograph, audio infrared and another sensor [6]. Accelerometer-based has several advantages, small, low computation, less expensive. With a small dimension as wearable devices or embedded in a smartphone, an accelerometer can use in the human body. The different position such as arm, waist, head, shoulder, pocket [20].

Popular public HAR dataset in UCI machine learning was used in several previous research [16]. This dataset was popular caused has three labeling, static activity (SA), dynamic activity (DA) and transitional postural activity (TA) very rarely a dataset that provides three labels at a time. Characteristic of this dataset each subject has sequence activities with a configuration of these three labels. However, this raises new problems solving confusing activities. In this paper, we purposed a simple linear method to solve confusing activities in efficient human activity recognition.

Section of this paper is organized as follows: Research Methods, provide information how this work was done, Data characteristic from HAR public datasets describe in Selection of data sets in Section 3, experimental result, model selection, testing models and at the last we will discuss and compare from previous research at end of this section. The conclusion is given in section 4 .

\section{Research Method}

\subsection{Human Activities}

Human activities such as walking, running, standing, laying can be defined as a set of action preformed by a user in a set of time on the environment [21]. Each activity can categorize by the complexity level in each recognition process. There are two categorized that several authors used simple or low-level categorized and complex or high-level complexity [22]. In low-level activities, for example, we can show walking, running, standing, sitting in these criteria and for high-level complexity driving, talking, working, shopping in this example.

Human activities recognition especially those that use sensors as data retrieval an instance of activity defined by the sequence of the sensor. Each activity has a sequence activity based on body pattern that sensor placement. In a set of activity that sequence of static activity to dynamic activity with a transitional position in each of both. For mathematic modeling, if we initialing sequence of activity with $A=\left\{a_{1}, a_{2}, a_{3}, \ldots, a_{n}\right\}$ where $a_{n}=\left\{e_{1}, e_{2}, e_{3}, \ldots, e_{n}\right\}$ with $e_{n}=($ time, sensor, label $)$ in this experiment we used triaxial accelerometer and three axial gyroscopes so we can rewrite $e_{n}=\left(\right.$ time,$\left\{a c c_{x}, a c c_{y}, a c c_{z}, g y r_{x}, g y r_{y}, g y r_{z}\right\}$, label). In this paper, we focused on solving the confusing activities.

\subsection{Method}

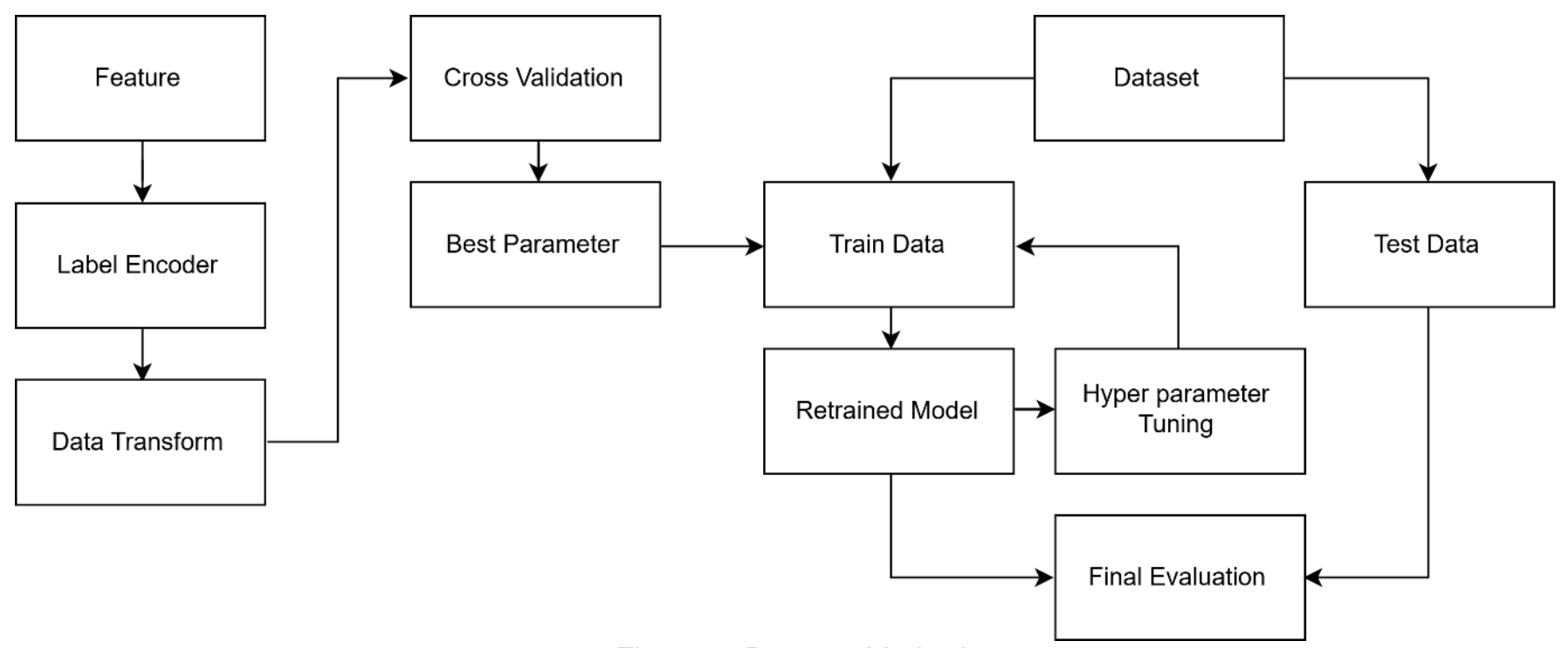

Figure 1. Purpose Method

In this paper Figure 1, we purposed linear method to solve confusing activities in public HAR dataset. In this public dataset has 563 features that are include subject and activities label in each row of data. We used all of the features without feature reduction and selection. Feature from HAR data set was labeling with label encoder and

(c) 2020 The Authors. Published by Universitas Muhammadiyah Malang

This is an open access article under the CC BY SA license. (https://creativecommons.org/licenses/by-sa/4.0/) 
transform to each row in data. We used several machine learning for comparation with the linear method was purposed in this paper. For each machine learning training the data up to three times. The best parameter in each training was used to predicting activities in testing data. For comparing with each machine learning method, we used accuracy and confusion matrix and with previous research we used accuracy.

\section{Results and Discussion}

In this section, we present the experimental result obtained in activity detection based on a public dataset. The performance was measured from accuracy and confusion matrix from several machine learning method. We first introduce training and dataset before we compare and analyze the recognition accuracy of several classifiers. In this paper, we verify the performance of the novel approach we purposed to improve LR with parameter tuning.

\subsection{Training and testing phase}

To evaluate classifier performance we used public UCI HAR dataset UCI HAR dataset [23] which is a renewal of the previous dataset [16]. Smartphone based dataset gathering from inertial sensor accelerometer and gyroscope. Six basic activities while carrying a smartphone attached to 30 participants belts. The datasets collected triaxial accelerometer and gyroscope with a constant rate of 50hz. Six basic activity based on tree static activity (SA) sitting, standing and laying, tree dynamic activity (DA) walking, walking up stairs, walking down stairs and six transition activity (TA) stand-to-sit, sit-to-stand, sit-to-lie, lie-to-sit, stand-to-lie, lie-to-stand. Every activity based on this tree phase sequent.

Label encoder and data transform were used in this paper, its significant increasing accuracy in each machine learning method. Data that was transformed in previous steps are used to train to get the best parameter in training steps. We used three training steps to get the best parameter in each machine learning as shown in Table 2, Table 3, Table 4, and Table 5. In both training and testing, we didn't use any feature selection or reduction. From 563 features in data set we just separated Subject and Activity.

\subsection{Dataset Characteristic}

Data from pubic HAR Dataset has 563 features, which has characteristic in postural transition label in each activity, the dataset was separated into data train and data set. Distribution of this dataset as shown in Table 1. Activity Data Distribution has configuration $70 \%$ data train which totals 7767 data and $30 \%$ data set 3162 data, there are no null data. This data set was gathering with the smartphone in a single position. The smartphone was located on the user's waist using a belt provided with a case [24]. With 30 subjects, they perform a series of movements that have been determined.

Table 1. Activity Data Distribution

\begin{tabular}{cccc}
\hline Activity Type & Activity & Train Data & Testing Data \\
\hline \multirow{3}{*}{ Static Activity } & Standing & 1423 & 556 \\
& Laying & 1413 & 545 \\
& Sitting & 1293 & 508 \\
\hline \multirow{3}{*}{ Dynamic Activity } & Walking & 1226 & 496 \\
& Walking Upstairs & 1073 & 471 \\
& Walking Downstairs & 987 & 420 \\
\hline & Stand To Lie & 90 & 49 \\
Transition Activity & Sit To Lie & 75 & 32 \\
& Lie To Sit & 60 & 27 \\
& Lie To Stand & 57 & 25 \\
& Stand To Sit & 47 & 23 \\
& Sit To Stand & 23 & 10 \\
\hline
\end{tabular}

Based on Ortiz et al [24] in each raw sensor signal from the accelerometer $\left(a_{r}(t)\right)$ and gyroscope $\left(\omega_{r}(t)\right)$ was preprocessed with series of filter for conditioning. The used thir-order median filter and third-order low-pass Butterworth filter for noise reduction. Frequency cut in $20 \mathrm{~Hz}$, based on human body spectrum in $0-15 \mathrm{~Hz}$. Data labels were defined between the end and the start consecutive static posture activity [23]. Each activity in this dataset start with SA then TA and at the end with DA this change activity sequence with scanned procedures. Data set from the experimental that was done by Ortiz et. al. [16] has relevant information regarding the activity groups. TA has $8 \%$ of the total data sets. However, TA has a limited duration which has $3.73 \mathrm{~s} \pm 1.2 \mathrm{~s}$ [23] every average duration in TA has slightly different. And now how to reduce the dimension of this data set and we will determinate data sets distribution. In this paper, we

Cite: Kusuma, W., Sari, Z., Minarno, A., Wibowo, H., Akbi, D., \& Jawas, N. (2020). Performance Comparisson Human Activity Recognition using Simple Linear Method. Kinetik: Game Technology, Information System, Computer Network, Computing, Electronics, and Control, 5(1). doi:https://doi.org/10.22219/kinetik.v5i1.1025 

used t-SNE which is a technique for dimensionality reduction that is suited to the visualization of high-dimensional data sets. We used TSNE in Sklearn method with 1000 iteration and perplexities 5, 30, 50, and 100.

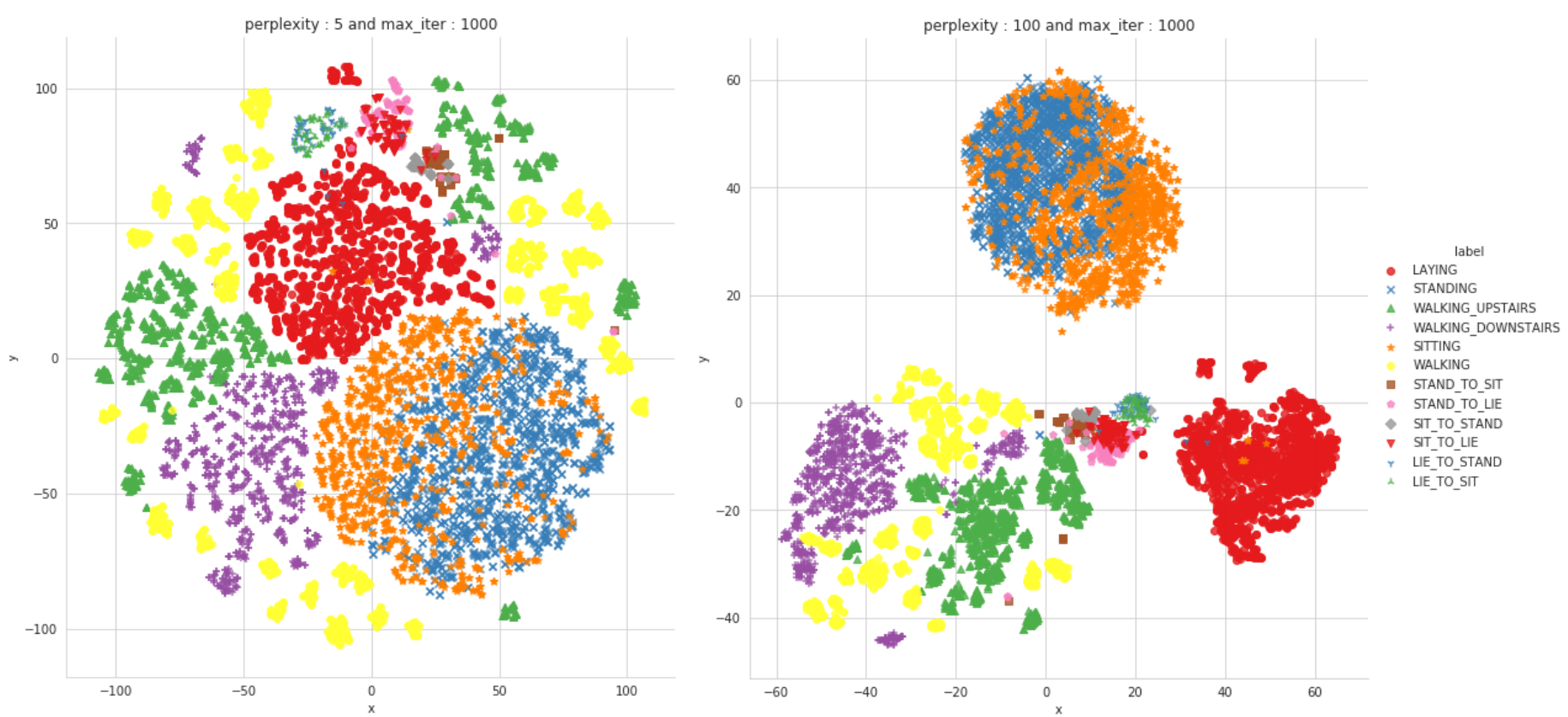

Figure 2. Data Dimensional Reduction

Figure 2 (Left) shown that at first iteration with perplexity $=5$ data slightly different with perplexity=100 in Figure 2 (Right). Both of them we can see that the digits are very clearly clustered in their own sub groups. If we would now use a clustering algorithm to pick out the separate clusters, we could probably quite accurately assign new points to a label. It is quite difficult to distinguish between sitting and standing even by increasing accuracy or perplexity.

\subsection{Machine Learning Comparation}

In this paper, we comparing several machine learning methods which have been widely used by several authors. For this paper, we used Logistic regression (LR), Support vector classification (SVC), Support vector machine (SVM) Decision tree (DT), Random forest (RF), Gradient boosting classifier (GB), and K-Nearest Neighbor (KNN). We will be comparing several methods with four steps, at first step we used SA, TA, and DA as shown in Table 2. ML Comparation of SA, TA, and DA. For every step, we used three phase training before used this model for predicting test data. SA, TA, and DA have sequent activity for each step. Predicting activity that used these three kinds of activity its more powerful to show this sequencing activity. However, for training and predicting time shown that it needs more times. With total up to six minutes for total training phase in all method. Accuracy from several methods shown that LR and SVC has a stable for training and accuracy.

Table 2. ML Comparation of SA, TA, and DA

\begin{tabular}{|c|c|c|c|c|c|c|c|}
\hline \multirow{2}{*}{ Method } & \multicolumn{5}{|c|}{ Training Phase } & \multicolumn{2}{|c|}{ Predicting Phase } \\
\hline & Time & & Accuracy & & Average Accuracy & Time & Accuracy \\
\hline LR & $0: 00: 15.318700$ & 0.97182555 & 0.97297297 & 0.973 & 0.972705463 & $0: 00: 00.009013$ & 0.945287793 \\
\hline SVC & $0: 00: 07.128817$ & 0.971 & 81467 & 7796 & 0.97257741 & 0:00:00.008625 & 4092 \\
\hline SVM & $0: 00: 13.573758$ & 0.91084523 & 91892 & 0.9 & 5799188 & $0: 00: 10.76$ & 0.918089817 \\
\hline DT & 0:00:06.815242 & 0.90968738 & 0.90772201 & 0.91376643 & 1941 & 0:00:00.005043 & 0.80740038 \\
\hline $\mathrm{RF}$ & 1.541489 & 0.944423 & & 0.9 & & & \\
\hline GB & I.122746 & 0.9729834 & 807 & $0 . \varsigma$ & 9870726 & 00 . & 0.919354839 \\
\hline KNN & $0: 00: 00.258442$ & 0.94635276 & 0.95135135 & 0.94895592 & 0.948886676 & $0: 00: 24.044030$ & 0.884882985 \\
\hline
\end{tabular}

Second steps, at this step we just used SA and DA label activity that's mean that we don't use all transition activities here. Even so, it's about differentiating static and dynamic activity that may we know that it is not possible to leave transition activity. Accuracy at this step increases compared to the previous stage, as shown in Table 3. ML Comparation of SA and DA for the training phase both LR and SVC reach $98 \%$ accuracy. Likewise, when the prediction gets an accuracy of $96 \%$, it showed a satisfactory number even though it was $2 \%$ lower than during the training.

(c) 2020 The Authors. Published by Universitas Muhammadiyah Malang

This is an open access article under the CC BY SA license. (https://creativecommons.org/licenses/by-sa/4.0/) 
Eliminating TA features increases accuracy and is faster than when we add TA features. Almost all methods produce pretty good accuracy, lowest accuracy at $85 \%$ for DT method.

In the second steps we have got very good results but it is not fair if we do not compare when using the TA feature with all activities for both static and dynamic. Table 4. ML Comparation of SA and TA and Table 5. ML Comparation of TA and DA has shown that we used TA for every activity but not used between activities as in the Table 2. ML Comparation of SA, TA, and DA. As was expected, the use of TA in each activity is no better than eliminating the feature. The highest accuracy when we used TA for SA in 93\%. However, some methods have decreased accuracies like an SVM and KNN. Likewise, when using TA combining with DA, the accuracy just $94 \%$ almost the same when we used SA, TA, and DA together.

Table 3. ML Comparation of SA and DA

\begin{tabular}{|c|c|c|c|c|c|c|c|}
\hline \multirow{2}{*}{ Method } & \multicolumn{5}{|c|}{ Training Phase } & \multicolumn{2}{|c|}{ Predicting Phase } \\
\hline & Time & & Accuracy & & Average Accuracy & Time & Accuracy \\
\hline $\mathrm{LR}$ & $0: 00: 06.004940$ & 0.98450245 & 0.98613377 & 0.9 & 0.984901614 & $0: 00: 00.006862$ & 95249 \\
\hline SVC & 0:00:04.015053 & $0.9 \varepsilon$ & 0.98 & & 37 & & \\
\hline SVM & $0: 6$ & 0.935 & 0.9 & 0.9 & 87 & & 78249 \\
\hline DT & $0: 00: 04.158879$ & 0.93066884 & 0.94575856 & 341503 & 46 & 67 & 0.857482185 \\
\hline $\mathrm{RF}$ & 1.053258 & & & & & & 78045 \\
\hline GB & .987060 & 0.98613377 & 0.98450245 & 78758 & 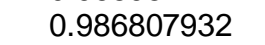 & 00.0 & 0.939260265 \\
\hline KNN & 0.232984 & 0.97063622 & 347 & 0.95996732 & 36567 & 392 & 0.90023753 \\
\hline
\end{tabular}

Table 4. ML Comparation of SA and TA

\begin{tabular}{|c|c|c|c|c|c|c|c|}
\hline Method & Training Time & & Accuracy & & Average Accuracy & Predicting Time & Accuracy \\
\hline LR & $0: 00: 04.983604$ & 0.95585284 & 0.95850067 & 0.95911528 & 0.957822931 & $0: 00: 00.005281$ & 0.933521127 \\
\hline SVC & 0:00:04.005604 & 0.95518395 & 0.95917001 & 0.96179625 & 0.958716736 & 0:00:00.013149 & 0.927323944 \\
\hline SVM & 0:00:06.065940 & 0.8729097 & 0.8875502 & 0.88538874 & 0.881949547 & 0:00:03.294605 & 0.898591549 \\
\hline DT & 0:00:02.258312 & 0.90434783 & 0.92034806 & 0.92091153 & 0.915202471 & $0: 00: 00.003311$ & 0.857464789 \\
\hline RF & 0:00:00.671379 & 0.93444816 & 0.94846051 & 0.94168901 & 0.941532559 & $0: 00: 00.006481$ & 0.903661972 \\
\hline GB & 0:02:05.953370 & 0.95852843 & 0.95515395 & 0.95844504 & 0.957375806 & $0: 00: 00.031060$ & 0.910985915 \\
\hline KNN & $0: 00: 00.114955$ & 0.92240803 & 0.91700134 & 0.92359249 & 0.92100062 & 0:00:08.556981 & 0.885633803 \\
\hline
\end{tabular}

Table 5. ML Comparation of TA and DA

\begin{tabular}{cccccccc}
\hline Method & Training Time & \multicolumn{3}{c}{ Accuracy } & Average Accuracy & Predicting Time & Accuracy \\
\hline LR & $0: 00: 02.788252$ & 0.98105437 & 0.98023064 & 0.97768595 & 0.979656986 & $0: 00: 00.004489$ & $\mathbf{0 . 9 4 2 6 9 1 5 6 5}$ \\
SVC & $0: 00: 02.217087$ & 0.98270181 & 0.98270181 & 0.98347107 & 0.982958233 & $0: 00: 00.004117$ & $\mathbf{0 . 9 4 9 1 3 0 7 1 5}$ \\
SVM & $0: 00: 03.925048$ & 0.92504119 & 0.92504119 & 0.92396694 & 0.924683105 & $0: 00: 01.831414$ & 0.90856407 \\
DT & $0: 00: 02.793941$ & 0.88962109 & 0.88632619 & 0.89669421 & 0.890880499 & $0: 00: 00.003351$ & 0.737926594 \\
RF & $0: 00: 00.613881$ & 0.94645799 & 0.92668863 & 0.93719008 & 0.936778902 & $0: 00: 00.007711$ & 0.849967804 \\
GB & $0: 02: 00.593826$ & 0.96952224 & 0.96952224 & 0.96198347 & 0.967009317 & $0: 00: 00.034825$ & 0.891822279 \\
KNN & $0: 00: 00.075742$ & 0.97116969 & 0.9645799 & 0.96694215 & 0.967563912 & $0: 00: 05.099911$ & 0.867353509 \\
\hline
\end{tabular}

From several comparations with any configuration was describe before we have known that LR and SVC have stable accuracy for any configuration with or without TA in training or predicting phase. SVC or SVM with the linear kernel is a method used for classification problems similar to LR. Before we describe why SVC and LR have stable and high accuracy in every testing, first we must show own configuration in every experimental. Data from data set was separated manually by an author with configuration $30 \%$ testing and $70 \%$ training. We were converting all non numeric features to numeric features with label encoding. A different number is assigned to each unique value in the feature column. A potential issue with this method would be the assumption that the label sizes represent ordinality. It seems that label encoding performs much better across the spectrum of different threshold values. However, there are a few features in which the label order did not make sense. In logistic regression, we take the output of the linear function and squash the value within the range of $[0,1]$ using the sigmoid function (logistic function). The Sigmoid-Function is an Sshaped curve that can take any real-valued number and map it into a value between the range of 0 and 1 , but never exactly at those limits. Typically, if the squashed value is greater than a threshold value, we assign it a label 1, else we assign it a label 0 . From the experiment, we can draw the conclusion that SVC tries to maximize the margin between the closest support vectors while LR the posterior class probability. Thus, SVC finds a solution which is as fare as possible for the two categories while LR has not this property. LR is more sensitive to outliers than SVC because the cost function of LR diverges faster than those of SVC. Having an outlier as shown in Figure 2. Data Dimensional Reduction more tolerable with LR. LR produces probabilistic values while SVC produces 1 or 0 . So in a few words, LR makes not an absolute prediction and it does not assume data is enough to give a final decision. This maybe is good

Cite: Kusuma, W., Sari, Z., Minarno, A., Wibowo, H., Akbi, D., \& Jawas, N. (2020). Performance Comparisson Human Activity Recognition using Simple Linear Method. Kinetik: Game Technology, Information System, Computer Network, Computing, Electronics, and Control, 5(1). doi:https://doi.org/10.22219/kinetik.v5i1.1025 
Kinetik: Game Technology, Information System, Computer Network, Computing, Electronics, and Control property when what we want is an estimation or we do not have high confidence into data. With used LR and SVC, we have known that this data set will be linearly separable with a simpler model.

\subsection{Comparation with previous research}

Previous research was introducing several machine learning methods to solve human activity recognition based on Table 6. Machine Learning Comparation we will be comparing with closer research.

Table 6. Machine Learning Comparation

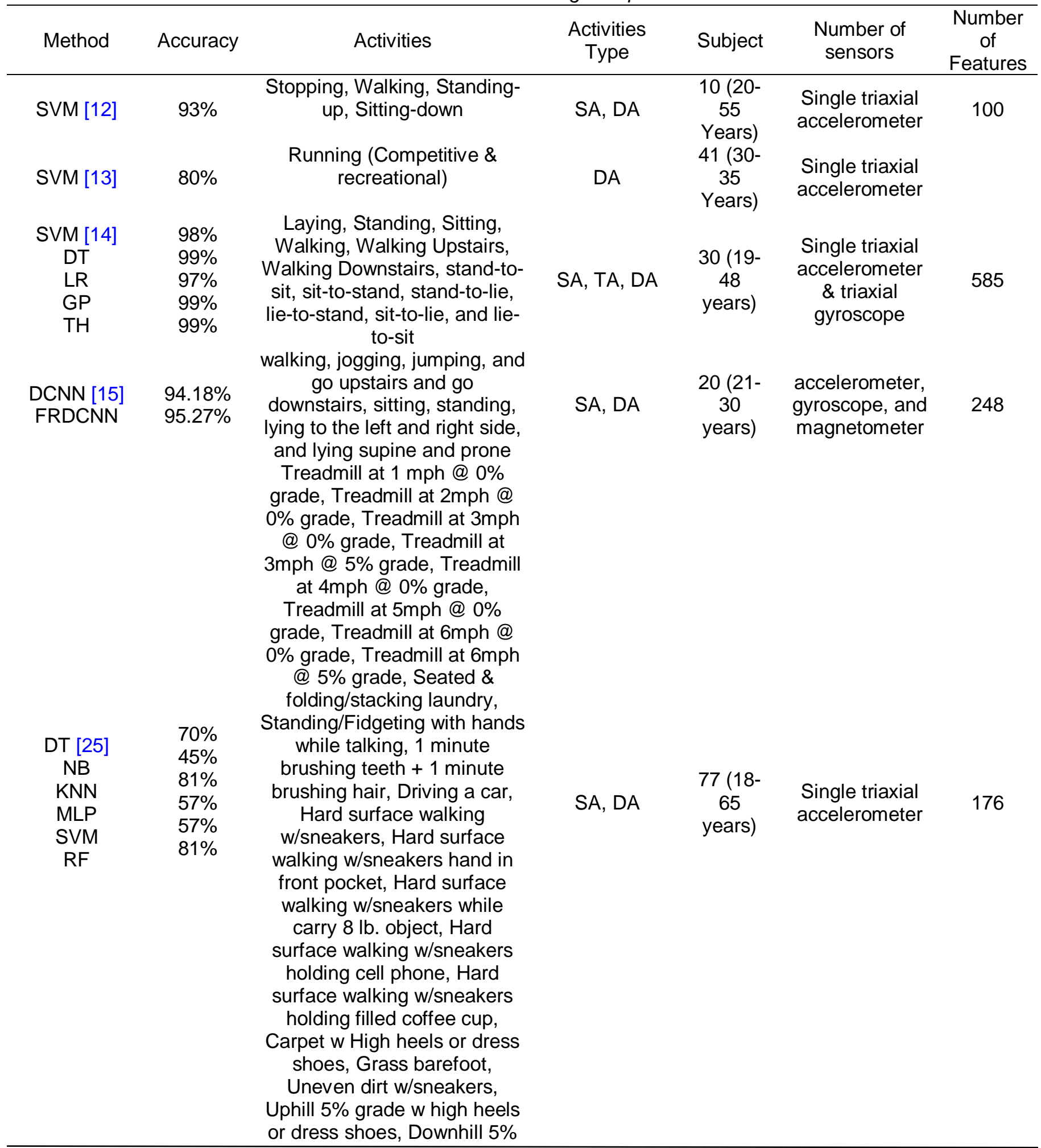

(c) 2020 The Authors. Published by Universitas Muhammadiyah Malang

This is an open access article under the CC BY SA license. (https://creativecommons.org/licenses/by-sa/4.0/) 
grade $w$ high heels or dress shoes, Walking upstairs $(5$ floors), Walking down stairs (5 floors)

Laying, Standing, Sitting, Walking, Walking Upstairs, SVM [16] 96\% $\quad \begin{aligned} & \text { Walking Downstairs, stand-to- } \\ & \text { sit, sit-to-stand, stand-to-lie, }\end{aligned}$ lie-to-stand, sit-to-lie, and lieto-sit

A Predictive algorithm 90

[26]

ANN [27]

$93 \%$

Euclidean Distance [17]

$95.8 \%$

Sitting, standing, drive

Walking, Running, Sitting, walking Upstairs, Downstairs, and Standing

sitting (duration $60 \mathrm{~s}$ ); 2. standing (duration $60 \mathrm{~s}$ ); 3. lying supine (duration $60 \mathrm{~s}$ ); 4 . sitting and talking (duration 60 s); 5. sitting and operating PC keyboard (duration $60 \mathrm{~s}$ ); 6 . walking (duration $60 \mathrm{~s}$ ); 7 . stairs up (duration about 40 s); participants were asked to climb stairs (60 steps) at their usual speed in the laboratory building; 8. stairs down (duration about $40 \mathrm{~s}$ ); and 9. cycling (duration about $40 \mathrm{~s}$ ); participants rode a bicycle around the block.

Walking, Walking carrying items, Sitting \& relaxing, working on computer, standing still, Eating or drinking, Watching TV, DT [18] $\quad 84 \% \quad \begin{aligned} & \text { Reading, Running, Bicycling, } \\ & \text { Stretching, Strength-training, }\end{aligned}$ Scrubbing, Vacuuming, folding laundry, lying down \& relaxing, brushing teeth, Climbing stairs, Riding elevator, Riding escalator standing, sitting, kneeling,

Pattern recognition neural networks [10]

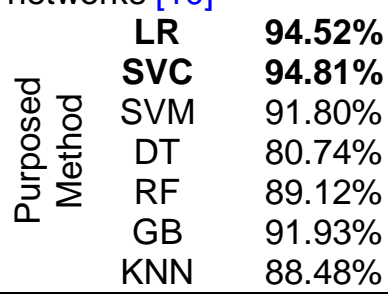
the face down, lying with the face up and lying on one side. Laying, Standing, Sitting, Walking, Walking Upstairs, Walking Downstairs
SA, DA

SA, TA, DA

$30(19-$ 48 years)

Single triaxial accelerometer \& triaxial gyroscope

$\begin{array}{ccc}\text { SA } & 1(42 & \text { Accelerometer } \\ & \begin{array}{c}\text { years }) \\ 10(\text { age }\end{array} & \\ \text { range } & \text { Single X, Y } \\ \text { not } & \text { Accelerometer } \\ \text { describe) } & \end{array}$

$24(21-$

Four Accelerometer Not describe Two biaxial accelerometers

Two Triaxial accelerometer 8

SA, DA $\quad \begin{gathered}34 \\ \text { years) }\end{gathered}$

SA, DA $\quad \begin{gathered}34 \\ \text { years) }\end{gathered}$

SA, DA range not describe)

SA, DA 4 (23-27)

$$
\text { SA, DA }
$$
$30(19-$
48

SA, TA, DA
Single triaxial accelerometer \& triaxial gyroscope 
In this paper we use a linear method such as LR and SVC, we separated in four step activity recognition, the best accuracy was reached in SA and DA and for other test has accuracy up to $94 \%$ as shown in Table 6. Machine Learning Comparation. According to the experiments presented in this paper we comparing several previous kinds of research. From this method with two approach model, selection and evaluated model. In this experiment SVM with RBF kernel has $91.80 \%$ accuracy. In previous research which used SVM as classified, Fuantes et. al. (93\%) [12] with the best accuracy for stop and walking activities. However, our accuracy is superior when using SVM with a linear kernel (SVC) with $94.52 \%$ also they used 100 related features such as angle calculation, the acceleration module, increments, and averages.

Fan et. al [14] (98\%) which used the same public HAR dataset and used SVM with the unknown kernel, but they have more features than as written by Anguita et.al. [16] that have 561 features, in [14] shown that used 585 features. However, both [14] or [16] have $98 \%$ accuracy they do more complex computing for feature selection and classification method and they used two classes, basic activity and transition activity that may more accurate result for recognizing static activity like standing and sitting. They also used LR with $97 \%$ accuracy slightly lower than the results we got $98.40 \%$ when we used SA and DA.

Bao et.al. [18] that used decision tree with $84 \%$ accuracy used 512 features with more than 20 activity almost the same as Kim et.al [25] that have 70\% accuracy but, Bao et.al. [18] used two biaxial accelerometers that are mean just have $\mathrm{X}$ and $\mathrm{Y}$. In another result that more comparable with this experiment was have been by Fan et. al [14] with $98 \%$ but with more 20 features than we used that recorded $93.44 \%$ with just 561 features. Although the results obtained not directly comparable with some previous studies. The purposed linear method has achieved efficient recognition and can be compared with several methods of deep learning including DCNN and FRDCNN that has 94.18\% and 95.27 accuracies [15] it is slightly lower than the results we got $98.40 \%$ with logistic regression or from another result from Euclidean distance with 95.8\% [17] or Pattern recognition neural networks with 99.8\% [10]. Figure 3. Comparation Confusion Matrix (a) SA and DA (b) SA, TA, DA has shown that its more reliable when used SA and DA than used TA in each activity.

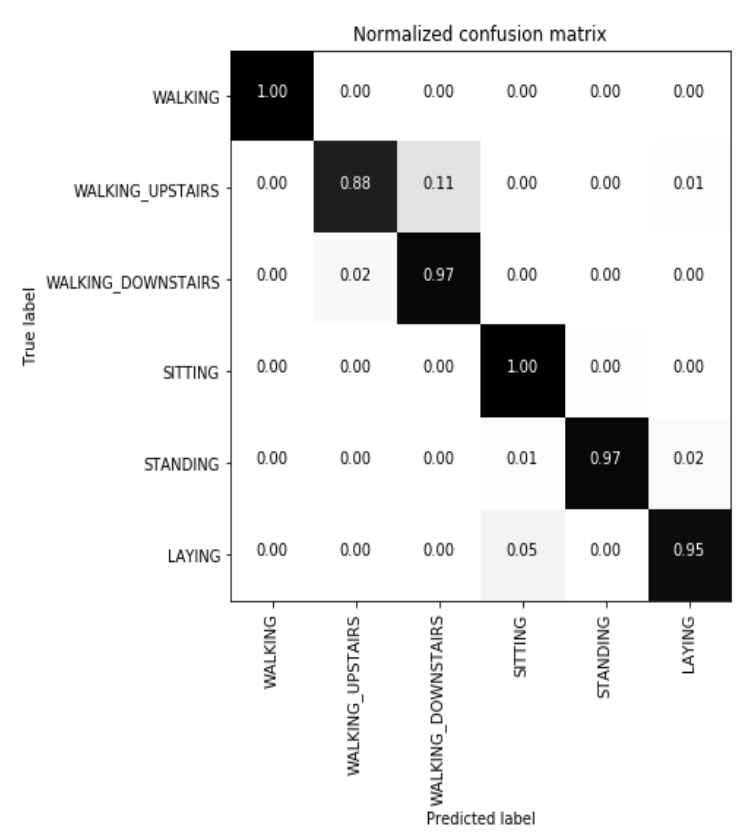

(a)

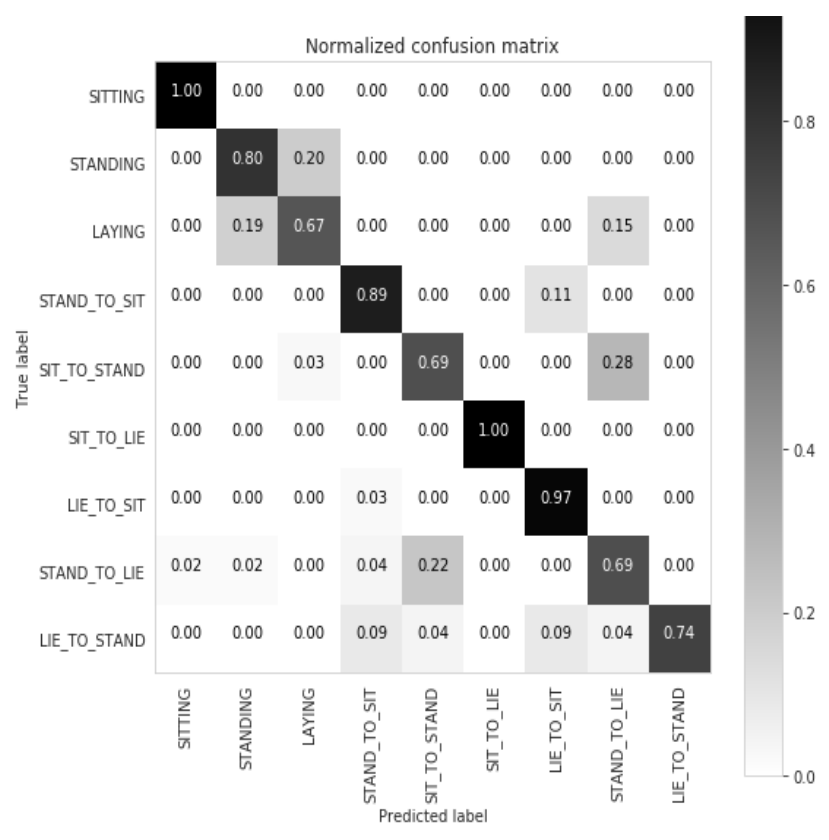

(b)

Figure 3. Comparation Confusion Matrix (a) $S A$ and DA (b) $S A, T A, D A$

\section{Conclusion}

This paper has purposed linear kernel based on human activity recognition model using a single position three axis accelerometer and three axis gyroscope data. We have compared and analyzed the performance of several algorithms with three criteria data static activity, dynamic activity, and transition activity. In order to further improvement in recognition accuracy data shown that it can linearly separable. With simple models like LR and SVC, we can reach an accuracy of up to $96 \%$ for both methods. However, transition activity may ambiguous data and it is shown that if we used transition activity in every activity both static and dynamic activity its accuracy will decrease. For future works, verify robustness of data and comparable with deep learning might reduce the features. In this paper, we used 561 features from 


\section{Acknowledgement}

Thank you to the Informatics Department and Informatics Laboratory, Universitas Muhammadiyah Malang which has become a place for researchers to develop this journal research. Hopefully, this research can make a major contribution to the advancement of technology in Indonesia.

\section{References}

[1] S. Shelke and B. Aksanli, "Static and dynamic activity detection with ambient sensors in smart spaces," Sensors (Switzerland), Vol. 19, No. 4, 2019. https://doi.org/10.3390/s19040804

[2] D. A. Fitriani, W. Andhyka, and D. Risqiwati, "Design of Monitoring System Step Walking With MPU6050 Sensor Based Android," JOINCS (Journal Informatics, Network, Comput. Sci., vol. 1, No. 1, Pp. 1, 2017. https://doi.org/10.21070/joincs.v1i1.799

[3] W. A. Kusuma, Z. Sari, H. Wibowo, S. Norhabibah, S. N. Ubay, and D. A. Fitriani, "Monitoring walking devices for calorie balance in patients with medical rehabilitation needs," Int. Conf. Electr. Eng. Comput. Sci. Informatics, vol. 2018-Octob, Pp. 460-463, 2018. https://doi.org/10.1109/EECSI.2018.8752761

[4] I. Gringauz et al., "Risk of falling among hospitalized patients with high modified Morse scores could be further Stratified," BMC Health Serv. Res., Vol. 17, Pp. 721, Nov. 2017. https://doi.org/10.1186/s12913-017-2685-2

[5] W. A. Kusuma and L. Husniah, "Skeletonization using thinning method for human motion system," 2015 Int. Semin. Intell. Technol. Its Appl. ISITIA 2015 - Proceeding, Pp. 103-106, 2015. https://doi.org/10.1109/ISITIA.2015.7219962

[6] O. C. Kurban and T. Yildirim, "Daily motion recognition system by a triaxial accelerometer usable in different positions," IEEE Sens. J., vol. 19, No. 17, Pp. 7543-7552, 2019. https://doi.org/10.1109/JSEN.2019.2915524

[7] C. Dobbins, R. Rawassizadeh, and E. Momeni, "Detecting physical activity within lifelogs towards preventing obesity and aiding ambient assisted living," Neurocomputing, vol. 230, No. February, Pp. 110-132, 2017. https://doi.org/10.1016/j.neucom.2016.02.088

[8] M. S. Tremblay, R. C. Colley, T. J. Saunders, G. N. Healy, and N. Owen, "Physiological and health implications of a sedentary lifestyle," Appl. Physiol. Nutr. Metab., Vol. 35, No. 6, Pp. 725-740, 2010. https://doi.org/10.1139/H10-079

[9] D. T. Villareal, C. M. Apovian, R. F. Kushner, and S. Klein, "Obesity in older adults: Technical review and position statement of the American Society for Nutrition and NAASO, the Obesity Society," Obes. Res., Vol. 13, No. 11, Pp. 1849-1863, 2005. https://doi.org/10.1038/oby.2005.228

[10] F. Ioana-Iuliana and D. Rodica-Elena, "Detection of daily movements from data collected with two tri-axial accelerometers," 2011 34th Int. Conf. Telecommun. Signal Process. TSP 2011 - Proc., No. 26, Pp. 376-380, 2011. https://doi.org/10.1109/TSP.2011.6043706

[11] C. D. Gómez-Carmona, A. Bastida-Castillo, J. García-Rubio, S. J. Ibáñez, and J. Pino-Ortega, "Static and dynamic reliability of WIMU PRO"M accelerometers according to anatomical placement," Proc. Inst. Mech. Eng. Part P J. Sport. Eng. Technol., Vol. 233, No. 2, Pp. 238-248, 2019. https://doi.org/10.1177\%2F1754337118816922

[12] D. Fuentes, L. Gonzalez-Abril, C. Angulo, and J. A. Ortega, "Online motion recognition using an accelerometer in a mobile device," Expert Syst. Appl., Vol. 39, No. 3, Pp. 2461-2465, 2012. https://doi.org/10.1016/j.eswa.2011.08.098

[13] C. A. Clermont, L. C. Benson, S. T. Osis, D. Kobsar, and R. Ferber, "Running patterns for male and female competitive and rec reational runners based on accelerometer data," J. Sports Sci., Vol. 37, No. 2, Pp. 204-211, 2019. https://doi.org/10.1080/02640414.2018.1488518

[14] S. Fan, Y. Jia, and C. Jia, "A feature selection and classification method for activity recognition based on an inertial sens ing unit," Inf., Vol. 10, No. 10, 2019. https://doi.org/10.3390/info10100290

[15] W. Qi, H. Su, C. Yang, G. Ferrigno, E. De Momi, and A. Aliverti, "A Fast and Robust Deep Convolutional Neural Networks for Complex Human Activity Recognition Using Smartphone," Sensors, Vol. 19, No. 17, Pp. 3731, Aug. 2019. https://doi.org/10.3390/s19173731

[16] D. Anguita, A. Ghio, L. Oneto, X. Parra, and J. L. Reyes-Ortiz, "A public domain dataset for human activity recognition using smartphones," ESANN 2013 proceedings, 21st Eur. Symp. Artif. Neural Networks, Comput. Intell. Mach. Learn., No. April, Pp. 437-442, 2013.

[17] F. Foerster, M. Smeja, and J. Fahrenberg, "Detection of posture and motion by accelerometry: a validation study in ambulatory monitoring," Comput. Human Behav., Vol. 15, No. 5, Pp. 571-583, Sep. 1999. https://doi.org/10.1016/S0747-5632(99)00037-0

[18] L. Bao and S. S. Intille, "Activity recognition from user-annotated acceleration data," Lect. Notes Comput. Sci. (including Subser. Lect. Notes Artif. Intell. Lect. Notes Bioinformatics), Vol. 3001, Pp. 1-17, 2004. https://doi.org/10.1007/978-3-540-24646-6_1

[19] O. D. Lara and M. A. Labrador, "A Survey on Human Activity Recognition using Wearable Sensors," IEEE Commun. Surv. Tutorials, Vol. 15, No. 3, Pp. 1192-1209, 2013. https://doi.org/10.1109/SURV.2012.110112.00192

[20] M. Yang, H. Zheng, H. Wang, S. McClean, and D. Newell, "IGAIT: An interactive accelerometer based gait analysis system," Comput. Methods Programs Biomed., Vol. 108, No. 2, Pp. 715-723, 2012. https://doi.org/10.1016/i.cmpb.2012.04.004

[21] W. S. Lima, E. Souto, K. El-Khatib, R. Jalali, and J. Gama, "Human activity recognition using inertial sensors in a smartphone: An overview," Sensors (Switzerland), Vol. 19, No. 14, Pp. 14-16, 2019. https://doi.org/10.3390/s19143213

[22] S. Dernbach, B. Das, N. C. Krishnan, B. L. Thomas, and D. J. Cook, "Simple and complex activity recognition through smart phones," Proc. 8th Int. Conf. Intell. Environ. IE 2012, Pp. 214-221, 2012. https://doi.org/10.1109/IE.2012.39

[23] J. L. Reyes-Ortiz, L. Oneto, A. Samà, X. Parra, and D. Anguita, "Transition-Aware Human Activity Recognition Using Smartphones," Neurocomputing, Vol. 171, Pp. 754-767, 2016. https://doi.org/10.1016/j.neucom.2015.07.085

[24] Y. Wang et al., Smartphone-Based Human Activity Recognition. 2017.

[25] Y. M. Kim, C. Theobalt, J. Diebel, J. Kosecka, B. Miscusik, and S. Thrun, "Multi-view Image and ToF Sensor Fusion for Dense 3D Reconstruction," Austrian Inst. Technol. GmbH, 2009. https://doi.org/10.1109/ICCVW.2009.5457430

[26] M. Uiterwaal, E. B. C. Glerum, H. J. Busser, and R. C. Van Lummel, "Ambulatory monitoring of physical activity in working situations, a validation study," J. Med. Eng. Technol., Vol. 22, No. 4, Pp. 168-172, 1998. https://doi.org/10.3109/03091909809032535

[27] C. Randell and H. Muller, "Context awareness by analysing accelerometer data," Int. Symp. Wearable Comput. Dig. Pap., Pp. 175-176, 2000. https://doi.org/10.1109/ISWC.2000.888488

Cite: Kusuma, W., Sari, Z., Minarno, A., Wibowo, H., Akbi, D., \& Jawas, N. (2020). Performance Comparisson Human Activity Recognition using Simple Linear Method. Kinetik: Game Technology, Information System, Computer Network, Computing, Electronics, and Control, 5(1). doi:https://doi.org/10.22219/kinetik.v5i1.1025 
